Human Ethology Bulletin - Proc. of the V. ISHE Summer Institute (2016): 4-29

Theoretical Review

\title{
Is Play AN EMotion? SOME ETHOLOGICAL OBSERVATIONS
}

\section{Glenn Weisfeld ${ }^{1}$ \& Carol Cronin Weisfeld ${ }^{2}$}

${ }^{1}$ Department of Psychology, Wayne State University, Detroit, Michigan, USA.

${ }^{2}$ Department of Psychology, University of Detroit Mercy, Detroit, Michigan, USA. ad4297@wayne.edu

\begin{abstract}
This paper begins with a list of commonly recognized forms of play and a discussion of definitions of play. This is followed by an overview of the ontogeny, phylogeny, mechanisms, and possible functions of human play. Next the question of whether or not play should be regarded as a separate emotion from interest is addressed. Play resembles the emotion of interest (or curiosity) in its affects and cognitive elicitors. Play differs from interest in exhibiting somewhat distinctive behavioral, visceral, and expressive emotional properties. This evidence, together with phylogenetic information, suggests that interest, exploration, and play constitute the same basic emotion, with exploration being an evolutionary offshoot of interest and play an offshoot of exploration.
\end{abstract}

Keywords: Play, ethology, function, emotion, interest, exploration.

\section{INTRODUCTION}

This paper explores the question of whether or not play constitutes a separate emotion or is a form of the emotion of interest (or curiosity). The paper begins with a suggested taxonomy, or ethogram, of play behaviors, and with proposed definitions of play. This is followed by an outline of the various facets of this family of behaviors: ontogeny, proximate 
causation, phylogeny, and - in a separate section - ultimate causation (Tinbergen, 1963). We then discuss how theorists address the questions of what an emotion is and what the basic human emotions are. This is followed by a section on similarities and differences between play and the emotion of interest, with a view toward addressing the question of the relation of play to interest. The last section contrasts play with exploration.

\section{TYPES OF PLAY BEHAVOIR}

Perhaps the first step in reviewing the literature on human play is to establish a typology, or ethogram, of categories of observable play. This will help us to define the behavior in question. List such as those of Aldis (1975) and Burghardt (2005) suggest that play can be categorized into commonly-accepted types such as these:

1. Locomotor: whole-body play that allows practice at balance and coordination

2. Object: manipulation and sensory examination of objects

3. Chasing: children prefer the chased role (LaFreniere, 2014b), suggesting that practice in evading attackers is the main benefit

4. Fighting, or rough-and-tumble play: the most-studied type of social play

5. Nurturance: often omitted from lists of play types but extensive in female primates

6. Sexual and Courtship Play: Sexual play, including mounting and thrusting, is common in mammals (Smith, 1984). Aldis (1975) posited a category of courtship play.

7. Pretend: prominent between 2 and 7 years of age, thought to enhance social skills

8. Language: children seem inherently motivated to babble and absorb language

The first two types are obviously solitary but can occur in a social context. The last two occur mainly in humans, although a captive chimpanzee was once observed to drag an imaginary pull toy (Hayes \& Hayes, 1951). Pretend play has been reported in captive chimpanzees, bonobos, orangutans and gorillas (reviewed by Cheney \& Seyfarth, 1990).

Burghardt (2005) mentions construction play.

Sedentary games and sports (which involve mock fighting and chasing) are sometimes regarded as falling within the realm of play.

Exploration is usually distinguished from play, as discussed below.

Young children play at some activities that actually constitute useful labor. For example, girls play with younger siblings but care for them at the same time. Children in traditional cultures typically regard this work as play (Gray, 2009).

\section{DEFINITIONS OF PLAY}

Burghardt (2005) wrote at considerable length about how to define play. His summary definition was that "Play is repeated, incompletely functional behavior differing from more serious versions structurally, contextually, or ontogenetically, and initiated voluntarily when 
the animal is in a relaxed or low-stress setting" (p. 82). This definition would seem to fit most of the categories of play above, especially play fighting. Gray (2009) described play as voluntary, intrinsically motivated, bound by mental rules, imaginative, and occurring when alert but not stressed. Burghardt maintained that play is generally not functional in its current context but instead has delayed benefits.

Most forms of play are observed mainly in immature animals for which play appears to provide practice in developing aptitudes of present and later utility. Sexual and courtship play, on the other hand, occurs mainly in mature animals and so seems to offer immediate adaptive payoffs, presumably enhancing the likelihood of copulation. Sexual and courtship play seems to fulfill the other criteria of play, being preliminary to "serious" sexual behavior and being pleasurable in its own right.

The criteria of Clark and Miller (1998) are similar to Burghardt's, but pertain specifically to children's play. These criteria include an "as if" quality characteristic of pretend play, spontaneity, pleasure, and flexible behavior. These scholars would seem to exclude sexual and courtship play; however, the latter form of play might indeed possess these elements if, for example, the lovers playfully feed, chase, or pretend to bite each other (Alley, Brubaker \& Fox, 2013).

\section{FACETS OF PLAY}

We might next analyze play by Tinbergen's (1963) four questions. But we need to recognize that play is really a family of behaviors with varying degrees of resemblance to each other. In the next subsections we will briefly describe the ontogeny, proximate causation, and phylogeny of play. This information is relevant to the question of the function, or ultimate causation, of play, which is treated in the next major section.

\section{Ontogeny}

Play is largely confined to the years before puberty in mammals. This suggests that play functions in large measure as preparation for behaviors at maturity. However, the benefits of play are sometimes immediately applicable as well. Play tends to be extensive in mammals with a repertoire of flexible behaviors that can improve with practice, and in species with prolonged parental care that delays the onset of independence from parents. The primates best exemplify this slow developmental strategy and exhibit the most play of any mammalian order.

Humans (Homo ludens) play throughout the life span, as do some nonhuman primates (Burghardt, 2005; Pellis \& Iwaniuk, 2000). Humans and other primates sometimes play with their offspring, but whether or not parents themselves benefit from these interactions is an open question. Perhaps parental play improves with practice, partly by being rewarded by feedback from the offspring when done well.

Children's play helps them to learn not only specific skills, including those particular to their culture, but also rule making. Piaget (1932) described how children's understanding of 
rules grows during development. Once they understand that rules may be altered through negotiation, older children manipulate, or play with, rule possibilities. This empirical attitude may endure in adults who explore theoretical and practical questions about natural phenomena.

In their investigations of natural phenomena, children seem to be guided, or constrained, by what evolutionary scholars refer to as folk biology, folk physics, folk psychology and the like (Geary, 2005). Just how play guides these primordial concepts would seem to demand more research.

\section{Proximate Causation}

Play is often accompanied by play signals, such as the primate play face and laughter, and the dog's play bow. Play signals indicate intention to play or to continue play. These emotional displays help to distinguish play fighting from angry aggression.

Social play is often regulated; that is, violation of the rules of play, such as rules of play fighting, are discouraged by the threat of angry retaliation. A child who plays too roughly may be punished or find the play bout terminated. More generally, various species use angry retaliation, or the rage reaction, to punish violators of social norms. "Rage" serves to punish animals that breach the tacit rules that keep dominance contests ritualistic and relatively harmless in many species, and rage can occur when one animal attempts to usurp another's prerogative of rank (Bernstein \& Gordon, 1974; Jensen, Call \& Tomasello, 2007). Vigorous aggression also frequently results from violations of territory, mating claims (Kummer, 1971), or possession of a food morsel (Clutton-Brock \& Parker, 1995; Kuester \& Paul, 1992). The field of psychology has been slow to recognize that violations of social norms are the preeminent elicitors of anger after infancy, rather than personal frustration (Fehr \& Fischbacher, 2004; LaFreniere, 2014a; Trivers, 1971; Weisfeld, 1972).

Initiation of play involves the limbic system, suggesting that play constitutes an emotional behavior. Panksepp (1998) emphasized the role of certain thalamic nuclei in reacting to the stimulus properties of objects. But the sensorimotor aspects of play require activation of extensive cortical regions and the cerebellum (Byers \& Walker, 1995). Interestingly, a brain region involved in processing odors, the posterodorsal part of the medial amygdala, was smaller in male rats housed individually and not allowed to play (Cooke, Chowanadisai \& Breedlove, 2000). This brain area may have remained undeveloped because of social isolation or reduced exposure to body odors, and not the absence of play.

\section{Phylogeny}

Aldis (1975) confined himself to studying play in various captive mammals, and play occurs disproportionately in mammals. However, play has been reported at least anecdotally or in captivity in numerous vertebrates of all Classes. In his review, Burghardt (2005) asserted that play is well established among invertebrates only in octopuses, which will play with 
Legos or play catch with balls by using their jets. His treatment of avian play is especially extensive.

Correlates of play include adequate metabolic energy, parental care to allow time and opportunity for play, and behavioral complexity that requires extensive learning. Play is prominent in primates, which are highly altricial, energetic, and behaviorally complex. Folivorous primates need more time to forage and to acquire metabolic energy; they tend to play less than frugivores and ominvores. Food shortages require conservation of metabolic energy and can diminish play. Aquatic mammals play a lot; their buoyancy reduces energy expenditure in play. Small mammals play less than large ones because small animals need to conserve energy for warmth. Predatory mammals, having more complex behavioral repertoires than prey species, generally play more. Humans may still play as adults because their behavior remains flexible.

The fact that chimpanzees play with objects and fashion tools (McGrew, 2010) but bonobos do little of either in the wild is difficult to explain in terms of these preconditions. Bonobos in the wild have access to potential play objects and tools, and are altricial, energetic, and behaviorally complex. Under semi-captive conditions, sanctuary-observed bonobos were observed to fashion and use tools to retrieve food (Roffman et al., 2015).

\section{ULTIMATE CAUSATION}

\section{General Cortical Development}

Facts such as these concerning the ontogeny, mechanisms, and phylogeny of play have led researchers to identify possible functions of play. Play would seem to be important in our species, in that hunter-gatherer children engage in it very frequently by modern societal standards (reviewed by Gray, 2009). Researchers seem to agree that play functions to enhance vestibulomotor and perceptual development, hence the concentration of play before maturity. Play and other forms of sensorimotor stimulation enhance cortical development in immature animals. Initially, play movements, like walking, are jerky, tentative, and clumsy, but become increasingly smooth, accurate, and economical. Thus, play at first resembles the rigid movements of reptiles, but becomes increasingly like the smooth, variable movements of mammals. Play may be extensive in mammals because only they possess a neocortex and a concomitant capacity for highly flexible motoric behavior and perceptual discrimination. Play is quite elaborate in some birds, especially the Corvinae, which exhibit highly flexible foraging and other behaviors (Burghardt, 2005). Ravens engage in acrobatic social games and play keep-away (Heinrich \& Smolker, 1998).

Much of the enjoyment of play comes from its novelty and variety. This is especially true of social play, including games. This variety ensures that the individual will practice a range of play behaviors. The impulse to play may therefore have been selected for to ensure that childhood motoric and social experience is sufficiently broad; more "serious" activities may not offer sufficient variety to develop musculoskeletal function of all body parts and movements, and social interactions in a variety of contexts. 


\section{Practice at Specific Behaviors}

Particular forms of play seem to serve narrower, more specific adaptive ends, such as predatory play in carnivores. Play chasing seems to improve avoidance of capture and shows little sex difference in children (Smith \& Connolly, 1980), unlike play fighting. Language play, starting with babbling, aids in production of speech sounds via auditory feedback. The evidence for these specific functions consists mainly of observations that individuals that engage in the mature behavior in question, such as predation, exhibit extensive play practice of that behavior. The evidence is mainly anecdotal but the general functional claim seems sensible. For example, Yanomami and Eipo children shoot play arrows (Eibl-Eibesfeldt, 1989), and Inuit children play with miniature dogsleds (Flaherty, 1922). Animals that exhibit a complex, specialized behavior would need to practice it, and play provides such an opportunity.

Pellegrini and Smith (1998) argued that the existence of the developmental sequence of rhythmic play, exercise play, and play fighting implies that each of these forms of play has a distinct function. They suggest that each form provides domain-specific experience for development of some particular neuromuscular capacity. Indeed, rhythmic movements of a particular set of muscles seem to increase just before voluntary movement of those muscles (Byers \& Walker, 1995).

Alternatively, brain development might be less rigid and more domain general, such that earlier, simpler experiences provide an epigenetic foundation for later, more complex developmental processes. Rhythmic, stereotypic play is certainly simpler than exercise play, which is simpler than play fighting. However, consistent with Pellegrini and Smith, rhythmic play may function to enhance the development of simpler bodily and manipulative movements, exercise play the development of complex coordination and balance, and play

fighting the development of combat skill. (Pellegrini and Smith assert that evidence for this last function is largely absent.) These views are not mutually exclusive.

It may be important to note that the capacity for performing skilled movements is not only cortical. Even without practice that would involve the cortex, Syrian hamsters shell seeds very quickly and skillfully (Schneider, 2014). And the corpus striatum is involved in the acquisition of habitual movement patterns. The thalamus and cerebellum are also involved in skilled movements.

\section{Maintaining Physical Fitness}

Some theorists have suggested that play helps to maintain muscles and cardiac output. Vigorous play leads to gains in physical fitness, including muscle tone and joint flexibility (Smoll \& Schutz, 1985). But these effects seem to be short-lived. When muscles are not used, as when a person breaks a leg, they quickly lose mass - atrophy of disuse. So play in childhood is unlikely to carry over to maintain the musculoskeletal system and stamina in adulthood. Play doubtless helps maintain physical fitness in the present, in childhood or adulthood, although other normal behaviors might suffice for this. 
Play also probably enhances long-term coordination, balance, specific motoric skills, and economy of movement. Play may be frequent throughout childhood partly because the body is changing size and bodily dynamics are in flux (cf. Thelen, 1995). Exercise play is frequent when the limbs grow rapidly during the preschool period (Tanner, 1970). However, this would not explain the increase in play during the juvenile growth plateau, or the decline in play during the adolescent growth spurt. Moreover, the notion that young adolescents experience an "awkward phase" during which they are discombobulated by the allomorphic growth of their limbs, makes no adaptive sense. Natural selection would not have tolerated a developmental stage of malfunction.

\section{Determination of Dominance Ranks}

Aldis (1975) and others have proposed that play fighting provides practice for later fighting for dominance. This would account for males' exhibiting more frequent and more years of play fighting than females due to greater prenatal exposure to androgens. Aldis doubted that play fights allow animals to sort out their dominance ranks, mainly because these encounters are friendly, with role reversals and mock attacks. However, Pellegrini and Smith (1998), as well as Blurton Jones (1972) and others, have argued that children can assay each other's strength during these play encounters. Pellegrini and Smith compared the restrained attacks of play fighting with ritualized, or the regulated, dominance contests of many species that sort out dominance relations with minimal injury. And just because friends often play together (Smith, 2010) does not mean that they do not establish a dominance relationship; friends tend to be close in rank but not identical in rank.

At any rate, children begin to identify each other's dominance rank starting around the time when play fighting begins, at three or four years of age (Omark et al., 1975). Over the next few years they gradually increase their agreement about who ranks where in their group. At this age boys are preoccupied with being "tough," by which they mean fighting ability. Stable dominance hierarchies are established by about six years of age. This dominance behavior is important not only in providing practice for dominance interactions later, but in its own right. Dominant girls as well as boys gain material and social prerogatives well before reproductive maturity; dominance is not just about male mating competition. Moreover, boys' dominance ranks exhibit surprising stability, on the order of 0.70 correlations for intervals up to 11 years, from age 6 years to 17 (Weisfeld et al., 1987). This means that the bodily changes of male puberty do not influence dominance ranks very much; boys who were dominant in childhood remain dominant, popular leaders in adolescence. Dominance relations among boys do seem to intensify at adolescence (Neill, 1985), probably because of the emergence of mate competition, but ranks remain stable in stable groups.

\section{Fostering Creativity}

Play in adult humans may help to generate novel ideas by prompting investigation and experimentation. Furthermore, play is often characterized by "out of the box," divergent 
thinking and therefore may enhance creativity and innovation (Bateson \& Martin, 2013). Testing this sort of proposition has been difficult. Play does seem to enhance subsequent cognitive performance, but apparently just by providing a respite from cognitive activity. The amount of exercise play does not seem to affect cognitive performance; only a break from classroom studying does (Pellegrini, Huberty \& Jones, 1995). It has been suggested that innovative ideas would have been rare events in prehistory and therefore creativity could not have been selected for. However, even a rare event might have been favored if it conferred a substantial adaptive advantage; like the law, natural selection grinds slow but exceedingly fine.

\section{Social Skills}

The ubiquity of pretend play in foragers (Smith, 2015) suggests that open-ended, variable play may be beneficial in the development of social understanding and social bonds. Studies of wild monkeys have shown that play facilitates peaceful interaction with strangers (Antonacci et al., 2010). Gray (2011) asserted that play helps a child to learn to take decisions, exert self-control, follow rules, and make friends. He argued that play, because it requires voluntary participation and fair treatment, cultivates egalitarian values and counterbalances dominance competition; the play of hunter-gatherer children is highly cooperative and age-mixed.

Evidence ties opportunities for pretend play to development of various social skills, including perspective taking (Lillard et al., 2013), as Piaget (1932) believed it did. Children's theory-of-mind scores have been found to be correlated with number of siblings with whom to interact (McAlister \& Peterson, 2006). Children seem to prefer true stories to fables, suggesting that realism, or ecological validity, is useful to them (Barnes, Bernstein \& Bloom, 2015). Smith (2010) pointed out that autistic children exhibit little pretend play and show theory of mind deficits, so pretend play may enhance that capacity or depend on it.

\section{Summary of Analysis of Ultimate Causes}

Some of these putative functions seem to be fulfilled in all cultures, such as development of motor skills and maintenance of physical fitness. The same may be true of determination of dominance ranks, since dominance emerges even in the assiduously noncompetitive Hopi (Weisfeld, Weisfeld \& Callaghan, 1984). All cultures seem to exhibit pretend play, which may aid in learning about adult roles. But cultures vary in the social context of play, toys provided to children, and the relation of play to work activities.

Gaskins, Haight, and Lancy (2007) explored some of these cultural differences by analyzing play in three cultural groups. In modern Western societies and Taiwan, play by children is actively cultivated by adults. Pretend play is encouraged and peaks early, between 3 and 6 years of age. Adults often provide toy animals and other such surrogates, and take roles in pretend play themselves. This extensive, and often fantastic, pretend play may help to expand children's ability to imagine hypothetical possibilities. Object play is extensive, 
abetted by the availability of toys. Much play occurs between peers, which may serve to help develop competitiveness.

Among the Kpelle, play is tolerated by not cultivated by adults. Pretend play occurs but is more realistic than that of the first group. Kpelle children engage in relatively simple play activities, consonant with the simpler work skills required in that culture. Object play is less complex. Young children at play are usually tended by older girls to ensure their safety. Older children perform work activities under the supervision of still older children.

Yucatec Mayan adults limit children's play in favor of encouraging work activities. Play is usually confined to the household compound. Pretend play, toys, and object play are quite limited. Pretend play peaks between 6 and 8 years of age. Older children supervise the younger ones, and must protect the adult community from interference from their charges.

Like so many other human behaviors, play is highly flexible and varies across cultures, genders, and individuals. The functions of play, therefore, vary somewhat, but can often be identified.

\section{SOME DUBIOUS FUNCTIONAL EXPLANATIONS}

\section{Energy Release}

Some functional explanations for play are problematic on theoretical grounds. These explanations are often suggested by people unfamiliar with evolutionary principles. Sometimes play is said to provide release of excess energy. But organisms evolve to conserve energy, not to expend it unnecessarily. The fact that many calories are expended by primates in play does not mean that this is the function of play; to the contrary, this is a cost of play. The law of economy, well known around 1900, is sometimes ignored in view of the benefits of moderate exercise in maintaining muscle strength, balance, agility, cardiovascular health, and cognitive function.

\section{Thermoregulation}

Play does indeed generate bodily heat if one is cold. However, shivering accomplishes the same thing. Furthermore, play (and shivering) require that blood be supplied to the muscles, whereas heat is retained by effortlessly diverting blood to the internal organs.

\section{Relief of Boredom}

A similarly dubious assertion is that animals play in order to relieve boredom. This transposes cause and effect. Emotions function to prompt adaptive behaviors. Adaptive behaviors do not evolve in order to make animals feel better. We do not eat in order not to feel hungry; we feel hungry so that we will eat. 


\section{Relief of Stress}

Similarly, animals do not play in order to relieve stress. If physiological reactions to stressors were generally maladaptive, they would have been selected out. If these reactions are generally adaptive, as surely they are, they could not be played away or smiled away. Furthermore, since conspicuous play can attract predators, awareness of this risk may increase feelings of stress concomitantly.

\section{Learning to Decode Emotional Expressions}

Pellegrini and Smith (1998) regard this as another dubious functional explanation for play. They note that boys play more than girls, yet females exceed males in nonverbal communication abilities (Hall, 1984). Also, many animals seem to understand their speciesspecific emotional expressions without engaging in play. One might add that, just as the universal facial expressions have an evolved basis, so must the ability to perceive these expressions accurately. In fact, the ability to respond appropriately to universal facial expressions appears in the first months of life and does not seem to require learning (Campos \& Barrett, 1984). Experience may, however, improve the ability to discern grades of these expressions, individual differences in expressions, blends of expressions, and expressions from various angles.

\section{COSTS OF PLAY}

The most obvious cost of play is time and energy expenditure. Pellegrini, Horvat and Huberty (1998) studied play in children ages 7 and 10; outdoor play was more energetically costly than indoor play, and play consumed about $6 \%$ of the children's energy budget. Play tends to decrease with food shortages in primates (e.g., Lee, 1984). Less obviously, the energy expended in primate play comes at the cost of growth during the juvenile period. The unique primate life history trait of slow juvenile growth has been attributed to the extraordinarily high amount of play in this arboreal order (Janson \& van Schalk, 1993). The primate growth spurt then compensates for this lost time in reaching full adult size.

In primates generally, play increases the risk of predation and of falling. A male infant howler monkey fell into a river and drowned during a play bout (Clarke \& Glander, 1984). Falls during play are a major cause of injury in children (Sacks et al., 1989). Injuries can occur in solitary or social play. Males are especially at risk of injury at play (Rivara et al., 1982). Children also risk suffocating from aspiration of small toys (Mofenson \& Greensher, 1985).

Given the particular risk of play in primates, with falls from trees common and great energy expenditure needed for locomotion in trees, it is remarkable that play is so very common in these mammals. It is likely that gains in learning during the protracted juvenile period compensate for these costs (Janson \& van Schalk, 1993. 


\section{DEFINING AN EMOTION}

In order to address the question of whether or not play is an emotion, it is necessary to agree on the definition of emotion and on the criteria for identifying the basic emotions. Unfortunately, agreement on the definition of emotion seems limited to a consensus that definitions of emotion abound. Kleinginna and Kleinginna (1981) found almost 100 definitions in the literature, and that was 35 years ago.

How is emotion defined? Many theorists, such as Scherer (1993), agree that an emotion is a behavioral complex that includes several facets: elicitors (internal and external), an affect, a behavioral tendency, and for some emotions a distinct emotional expression or pattern of visceral changes (Sander, 2013). Some theorists include an appraisal process for identifying emotionally salient elicitors, but emotions can be experienced even in the absence of consciousness of the elicitor or any cortical involvement (Zajonc, 1984). Which of these facets of an emotion should serve as the main indicator of a basic human emotion?

Paul Ekman defined an emotion by its exhibiting several properties: "automatic appraisal, commonalities in antecedent events, presence in other primates, quick onset, brief duration, unbidden occurrence and distinctive physiology [by which he meant neural mediation]" (1994, p. 18). Like Panksepp (1998), Ekman doubted that there are any human emotions not found in other species. Ekman (1994) proposed a list of 17 emotions that he suggested meet those requirements, including interest and sensory pleasure. Certainly these properties help us to identify what is meant by an emotion. However, reflexes would seem to meet these criteria also, with the possible exception of appraisal.

\section{IDENTIFYING THE BASIC HUMAN EMOTIONS}

A closely related question is how to identify the basic human emotions. Various attributes have been employed for this; some will now be considered.

\section{Neural Mediation}

As for the neural mediation of particular emotions (Ekman's "distinctive physiology"), controversy exists as to whether or not any such specificity can be demonstrated (Lindquist et al., 2012). It seems that certain limbic structures do specialize in mediating particular emotions, such as the anterior insula for disgust and the lateral hypothalamic area for hunger (see reviews by Phan et al., 2002; Murphy et al., 2003; Hamann, 2012). However, a given structure typically mediates more than one emotion; for example, the insula also receives gustatory, olfactory, fear, and pain input (Mohanty \& Gottfried, 2013; Rainville, 2013). As more is learned about specific nuclei in various limbic structures, doubtless more specificity will come to light. Neural mediation offers a promising tool for reaching consensus on the basic emotions.

Another way of approaching the question of identifying the basic emotions is by the facets of an emotion listed above: elicitors, affect, behavior, expression, and visceral changes. How promising are these facets for identifying a basic emotion? Interestingly, Ekman used 
only elicitors ("common antecedent events") as an indicator of a basic emotion. He did not include expression or affect. The next subsections will examine each of these facets as candidates for identifying the basic human emotions.

\section{Emotional Expressions as Indicators of the Basic Emotions}

In the wake of Ekman's work on universal facial expressions, many researchers began and continue to define an emotion as manifesting a specific facial expression, namely the six identified by Ekman and Friesen (1971) as being sent and received in a common way in different cultures. Defining emotions in terms of their observable expressions has inherent appeal to ethologists with their interest in observable behavior and animal displays.

However, facial expressions have limitations as indicators of the basic human emotions. Ekman (1994) readily acknowledged that some basic emotions lack a specific facial expression: "A universal signal should not ipso facto be considered evidence of an emotion. Nor should the lack of a universal signal be used to say a phenomenon is not an emotion" (p. 18). In fact, some commonly recognized emotions lack a distinctive facial expression, such as love, sexual feelings, and hunger. Ekman (1994) doubted that interest has a distinct facial expression. Some emotions are characterized not so much by a facial display as by a bodily display, such as pride and shame (Tracy \& Robins, 2008) or a vocal display, such as mirth (Provine \& Yong, 1991). Moreover, the facial expressions of joy and sadness reflect different forms or sources of pleasure and displeasure, and so are not good indicators of basic human emotions (Ekman, Davidson \& Friesen, 1990). Various affects can give rise to the same expression; for example, we may laugh out of amusement, nervousness, or social convention. In light of these limitations, we believe that emotional expressions are a poor candidate for indicators of the basic emotions, although doubtless they offer some useful evidence.

\section{Visceral Changes}

What about visceral changes? Controversy has always surrounded the question of the specificity of these patterns, starting with Cannon (1927). A recent review indicates that there is only modest specificity for visceral patterning, with numerous contradictory results and replication failures (Harrison, Kreibig \& Critchley, 2013). Two emotions can give rise to similar visceral changes, and a given emotion can prompt different visceral patterns in different individuals. Moreover, visceral changes are minimal in some emotions, such as drowsiness and interest.

\section{Affects}

Other theorists refer to facets notably absent from Ekman's criteria, especially affect and behavioral tendency. Panksepp (2005), for example, emphasized the consistent links between a particular affect and a resulting specific behavioral impulse, such as hunger and feeding: "Indeed, there seem to be as many distinct internally experienced affective states as there are basic motivational and emotional systems of the brain" (1994, p. 396). Affect may be the best single criterion for a basic emotion. Lang (2010, p. 229) asserted that "Scientists 
and laity agree...that emotions are inner feelings.... Affect is doubtless the most distinct feature of an emotion complex; all the other facets are found in non-emotional behaviors: Patterns of visceral changes occur in exercise and digestion; observable behavior characterizes reflexes; and elicitors prompt neutral sensations. Definitions of emotion that exclude affect are vacuous and unsatisfying.

Self-reports of affects are difficult to quantify and suffer from other methodological limitations, such as social desirability effects and the notorious imprecision of terms referring to emotional states. However, self-reports can be correlated with more objective neurological measures. For example, scores on the Beck depression scale have been correlated with right insula activation when subjects watched a film clip (Farb et al., 2010), and reports of olfactory preferences for body odors of same-sex or opposite-sex individuals are correlated with activation of the anterior hypothalamus (Berglund, Linström \& Savic, 2006). Another technique is to ask subjects to match their feelings with photos depicting particular emotional expressions (Chapman et al., 2009). Despite their methodological limitations, self-report data on affects provide an essential window into emotional experience.

\section{Behavioral Forms}

What about the behaviors prompted by the various affects? We primates exhibit great behavioral flexibility, so that many actions can stem from the same affect. Even in mammals generally, fear can give rise to flight, freezing, hiding, death feigning, and defensive attack. To define the basic emotions by their characteristic observable behaviors would seem to result in an ungainly number of basic emotions. However, observable behaviors can provide some indication of a basic emotion despite our behavioral flexibility; we ingest food by means of forks, hands, and chopsticks, but in all cases we convey food to the mouth.

It is perhaps worth mentioning that relatively little research is conducted on observable emotional behaviors, especially under naturalistic conditions. Much more research is conducted on elicitors, expressions, and visceral changes in the laboratory. Still less research is conducted on how one person's observable emotional behavior or expression influences another person behaviorally. For example, under what circumstances does the play face elicit a playful response and when is it rebuffed?

\section{Integrating Various Sources of Information}

It seems that information about all these facets of an emotion and its neural mediation is useful, to varying degrees, in identifying the basic emotions. To this information might be added comparative research on behavior of animals in the wild. For example, ethological observation of various species suggests that the common distinction in the human literature between emotions and drives make little sense in terms of animal behavior. Animals in the wild feed, sleep, drink, fight predators, seek suitable habitats, compete for rank, nurture their young, mate, etc. - which of these behaviors are emotions and which are drives? Taking a functional view of the basic emotions helps to identify them; presumably, each species 
possesses just those emotions necessary and sufficient to satisfy its various fitness needs. Lists of basic emotions offered by evolutionarily oriented scholars reflect this comparative, functional perspective and show considerable consensus, as indicated by the following table (Tab. 1) of lists of some basic emotions recognized by notable biologically oriented theorists:

Table 1. Some lists of basic emotions. Based on McDougall (1923), Plutchik (1980), Ekman (1994), Pugh (1977), and deCatanzaro (1999).

\begin{tabular}{lccccc}
\hline & McDougall & Plutchik & Ekman & Pugh & deCatanzaro \\
\hline Tactile pain and pleasure & & & $\mathrm{X}$ & $\mathrm{X}$ & $\mathrm{X}$ \\
Hunger & $\mathrm{X}$ & $\mathrm{X}$ & & $\mathrm{X}$ & $\mathrm{X}$ \\
Thirst & & & & $\mathrm{X}$ & $\mathrm{X}$ \\
Smelling & & & & $\mathrm{X}$ & \\
Disgust & $\mathrm{X}$ & $\mathrm{X}$ & $\mathrm{X}$ & & $\mathrm{X}$ \\
Sexual feelings & $\mathrm{X}$ & $\mathrm{X}$ & $\mathrm{X}$ & $\mathrm{X}$ & $\mathrm{X}$ \\
Love & $\mathrm{X}$ & & $\mathrm{X}$ & $\mathrm{X}$ & $\mathrm{X}$ \\
Interest & $\mathrm{X}$ & $\mathrm{X}$ & $\mathrm{X}$ & $\mathrm{X}$ & $\mathrm{X}$ \\
Humor appreciation & $\mathrm{X}$ & & $\mathrm{X}$ & $\mathrm{X}$ & \\
Pride/shame & $\mathrm{X}$ & & $\mathrm{X}$ & $\mathrm{X}$ & $\mathrm{X}$ \\
Anger & $\mathrm{X}$ & $\mathrm{X}$ & $\mathrm{X}$ & $\mathrm{X}$ & $\mathrm{X}$ \\
Fear & $\mathrm{X}$ & $\mathrm{X}$ & $\mathrm{X}$ & $\mathrm{X}$ & $\mathrm{X}$ \\
\hline
\end{tabular}

Functional analysis of the human emotions is exemplified by the approach of Pugh (1977), who was an engineer by profession and thought in terms of what voluntary behaviors people would need to execute in order to meet all of their fitness requirements. This led him to recognize elimination behaviors as emotions, as well as emotions occurring only in infancy: a "rocking urge" and a "sucking urge" (distinct from the sucking reflex and the hunger motive). Emotional experience varies qualitatively and quantitatively over ontogeny, but most non-evolutionary theorists confine themselves to identifying the adult emotions. Since play occurs mainly in immature animals, this point is especially relevant to the question of whether or not play constitutes a distinct emotion, a topic to which we now turn.

\section{IS PLAY A DISTINCT EMOTION FROMI NTEREST?}

Play seems to meet some but not all of Ekman's properties of an emotion. Play is present in other primates and can occur "unbidden," but seems to lack automatic appraisal, specific neural mediation, commonalities in antecedent events, quick onset, and brief duration. 
These last three properties seem to be lacking because play usually arises spontaneously rather than being elicited by an external event. But if we insist on emotions being elicited externally, we would exclude many instances of hunger, sex, thirst, and other emotions. It is likely that all emotions can be elicited by both internal and external events; for example, one eats when hungry and when food is present. One must regulate food intake and feed opportunistically, but also meet particular tissue needs. An animal seeks out a play partner and also responds to play invitations. Similarly, animals show a need to play but indulge this need only when safe and satisfied. Ekman's criteria seem questionable for defining an emotion and therefore for determining just what the basic emotions are.

Of the theorists cited in the table, play was mentioned only by McDougall and deCatanazaro. Darwin (1998) did not mention it. DeCatanzaro described play in a separate section from his description of "curiosity and exploration." LaFreniere (2014b, p. 193) stated that "play appears to be a primary affective/motivational system," and Panksepp (2008) recognized playful joy and laughter as a distinct positive socio-emotional system. However, McDougall and many other theorists seem to fold play into the emotion of interest. So, is play separate from interest?

To address the question of whether or not play is distinct from interest, it may be helpful to examine the particular facets of play behavior and the extent to which these facets differ from those of the emotion of interest.

\section{Visceral Changes}

The visceral changes characteristic of play are those that enhance vigorous bodily exertion. The emotion of interest, by contrast, is characterized by slowing of the heart rate, at least when the individual is passively investigating an object. Also, interest (but not play) is characterized by pupillary dilation, perhaps to enhance visual examination of a dimly lit stimulus. By Darwin's principle of direct action of the nervous system, pupillary dilation during interest also constitutes an emotional expression (Schmehl \& Oberzaucher, 2014).

\section{Expressions}

The play face seems distinct from the expressions of interest but perhaps mainly insofar as play is social whereas a person can be interested in something while alone. The play face and laughter accompany social play but, like most emotional expressions, are primarily observed when a receiver is present. Play signals seem to occur mainly in play fighting to distinguish this activity from aggression (Burghardt, 2005). The existence of play signals in so many species suggests that play is indeed distinct from the emotion of interest. However, as discussed above, expressions are a weak criterion for identifying the basic emotions.

\section{Behavioral Forms}

The behavioral forms that play takes, like the elicitors of play, are exceedingly varied, manifesting themselves as various forms of examination and manipulation of objects with variegated physical properties. The same is true of the behaviors and elicitors of interest. 
Some categories of behavior, such as object manipulation, can be said to arise either from a desire to play or out of curiosity, so the boundaries between play and exploration are often blurry.

However, not all curiosity leads to play, as Hutt and Hutt (1965) remarked. Passive visual examination of stimuli is not usually regarded as play. Play and interest differ in that play is more often vigorous and interest is more often passive. Interest can in fact be said to characterize any behavior of emotional significance to the subject; our attention is drawn to fitness-salient stimuli (Izard, 1977). Thus, play entails interest but so do potential mates, dangers, foods, and a host of other elicitors of emotion. When these encounters are "serious," the emotion of play is absent; play is inhibited in such circumstances.

\section{Affect}

Outdoor play with peers is pleasurable and is generally preferred by children in various cultures over watching television, using electronic games (Singer et al., 2009), associating with adults, or solitary play (Csikszentmihalyi \& Hunter, 2003). The affect of play seems hard to distinguish from that of interest. Both activities are described as engaging and their absence as boring. Izard (1977, p. 216) described interest as including "a feeling of wanting to investigate, become involved, or extend or expand the self by incorporating new information and having new experiences with the person or object that has stimulated interest." The same might be said of play. Play is interesting, which suggests ipso facto that play is adaptive.

Interest shows deprivation effects, the experience of which is referred to as boredom, the negative end of the affect continuum for this emotion. Play too shows deprivation effects, suggesting that it is a specific adaptive emotion that arises out of an internal need. When a child has not played for a while, she may be said to be bored. When she has played sufficiently, she stops playing. The fact that the amount of solitary play is not correlated with the amount of social play suggests that they have different functions (Roper \& Hinde, 1978); if the amounts were negatively correlated, this would infer that one could compensate for the other. But to claim that solitary play and social play constitute separate emotions would open the way to claiming that each form of play constitutes a separate emotion-a case of reductio ad absurdum. It seems that children need to practice a range of activities through various forms of play, just as people need to consume a variety of nutrients.

\section{Elicitors}

The elicitors of play resemble those in interest in several ways. These elicitors, the stimuli that prompt or inhibit play or interest, do so by influencing some positive and negative affects associated with these behaviors. In general, novelty enhances the pleasure of play and likewise the interest value of stimuli (see discussion by Edwards, 1999). The appeal of novelty in play and curiosity (or exploration) allows the individual to learn new things, as for example in manipulating objects or switching between attack and defense in play fighting. It 
is actually moderate novelty that maximizes interest. Low novelty, or familiarity, provides little opportunity for learning. Extreme novelty tends to induce fear and therefore avoidance of play and exploration. Think of the "secure base" that a mother monkey provides to its infant.

Similarly, moderate complexity maximizes interest and play. Extreme complexity is aversive-unsolvable problems and impossible play maneuvers provide little opportunity for learning and need to be abandoned. Low complexity likewise does not aid learning - the task or play activity is too simple. The fact that moderate complexity maximizes interest is called the cognitive consistency, or cognitive congruity, principle. It also characterizes humorous content. Pellegrini and Smith (1998) included the rhythmic movements of infants in their categories of play. These movements increase in complexity with development, as described by Piaget (1932) in his "circular reactions" terminology. Also, solitary play generally emerges before more complicated peer play.

As children in traditional societies begin to perform useful labor, they often regard tasks such as fetching objects or tending pets as play. As these tasks become mastered and boring, they are passed on to younger children so the older child can undertake more difficult, challenging tasks. This developmental pattern maintains interest at all levels of task difficulty, thus ensuring that all tasks get performed competently.

When we solve a problem, we experience a positive affect, the "aha!" of insight. Similarly, when a child succeeds at a play task such as placing a round peg in a round hole or turning a somersault, she seems pleased. This is sometimes referred to as exemplifying mastery motivation. The pleasure of discovering a solution rewards us for a successful effort. But unrelenting failures are aversive, causing us to abandon a hopeless task.

Thus, fluctuations in the affects entailed in play and interest guide learning in adaptive ways. Note that mastery motivation, if defined as the pleasure of perceiving a solution, is distinct from pride, or achievement motivation, which comes later in development and so is different. Pride/shame is also different from mastery motivation in having a distinct universal expression involving gaze direction and bodily posture and movement (Tracy \& Robins, 2008).

We can also examine the extent of resemblance of play to interest by considering Tinbergen's facets of these emotions. Take ontogeny: play predominates during years of immaturity, whereas interest prevails throughout the life span-although children are especially curious and inquisitive. The neural mechanisms of play and interest are probably too poorly understood at present to offer much insight into the question of resemblance. The function of interest is to enhance acquisition of knowledge about many things, particularly matters of fitness relevance. Play also functions to drive information gathering, but especially information and skills concerning bodily movement, social interactions, and language. Phylogenetically, play occurs mainly in mammals but seems to have evolved elsewhere by convergent evolution many times. On the other hand, interest in certain stimuli rather than others appears to be a general characteristic of animals. 
Most theorists cited above have noted the close resemblance of play to interest. Play resembles interest in the characteristics of its elicitors (such as moderate novelty and complexity), its affect, its behavioral forms, and its functions. The parallels between interest and play suggest that they have some common evolutionary roots. Play can grade into interest, as in "playing" board games, cards, sports, a theatrical part, or a musical instrument (Bateson \& Martin, 2013), rendering the distinction nebulous.

It appears that play differs to some extent from interest with regard to visceral changes, expressions, ontogeny, and phylogeny, and so has been selected for specifically. Play motivation may have evolved in mammals as an extension of the more general emotion of interest. Play may provide for the variety of sensorimotor experience necessitated by the great behavioral flexibility of mammals. But since play motivation seems to share the same affective components as those of interest, play should probably not be regarded as a separate emotion. We believe that affect is the most characteristic feature of an emotion, so we give greatest weight to it. Play and interest also overlap considerably in their behavioral forms, another important point of resemblance from an ethological standpoint.

\section{IS PLAY DISTINCT FROM EXPLORATION?}

Where does exploration fit in? Exploration is often distinguished from play. An animal that is introduced to a new space will usually explore it before even feeding when hungry; its motivation seems to include an element of fear that is reduced by familiarizing itself with its new surroundings. An animal typically explores a new environment first, and then proceeds to examine and explore the properties of objects within that environment.

Burghardt (2005) distinguished between "exploration or curiosity" and play. Many if not all of the distinctions can perhaps be explained away by recognizing that exploration often precedes play. One distinction is based on context: novel in the case of exploration and familiar in the case of play. However, this distinction can be folded into another one, that of timing, in that exploration commonly occurs before play. Burghardt also claims that exploration and play differ in affect: neutral or negative in exploration and positive in play. This distinction can be explained by noting that exploration of a novel environment or object can induce fear, especially if the situation is unfamiliar. Once the animal is assured of safety, it can proceed to examine particular features in a more relaxed and pleasurable state, just as play tends to be released by conditions of safety. Exploration and play are said to differ with regard to heart rate: stable in exploration and variable in play. Well, play, especially locomotor and social play, is often vigorous, whereas exploration is less energetic. None of these distinctions seems very compelling or sufficient to justify regarding exploration and play as qualitatively different emotions, especially if one grants that exploration often entails an element of fear.

Other differences between play and exploration have been discerned. Burghardt (2005) pointed out that play, unlike interest, often involves play signals, and predominates before puberty. As mentioned above, Burghardt pointed out that exploration or curiosity is often 
unpleasant, whereas play is usually fun. Exploration and play differ in their expression, exploration being mainly solitary. Exploration and play differ in their motoric elements, play often being more vigorous. Exploration may be elicited more by external stimuli and play by an internal need. Play is more spontaneous and variable than exploration, as Burghhardt pointed out. Exploration seems to involve somewhat different neural structures than play: the lateral hypothalamus and preoptic region are active when rats explore, and elicit selfstimulation (Komisaruk \& Olds, 1968). But it is axiomatic that different behaviors entail somewhat different neural pathways even if they all utilize some of the same structures, such as the corticospinal tract (the "final common pathway") or the dopaminergic reward pathway, which appears to be active in various pleasurable states (Berridge \& Robinson, 1998).

Exploration and play differ somewhat phylogenetically. Fish, amphibians and reptiles show some evidence of exploration but they exhibit little play, presumably because of its energy costs (Burghardt, 1988). This suggests that exploration and play may be spurred by the same emotion but play requires more energy. It seems that the energy demands of play were met only by the homeothermic birds and mammals, and that play warrants extensive energy expenditure only in animals whose flexible behavior requires extensive practice to develop, the mammals.

One might conclude that exploration and play differ in some ways but share the same affect, assuming we tease apart the fear element in exploration. Both exploration and play seem to involve the affect of interest and boredom; one can be interested in one's surroundings or bored with them, and the same for any play activity. Both exploration and play exhibit the cognitive congruity principle: moderate complexity is maximally pleasurable.

If we adopt the criterion of affect as the main characteristic of an emotion, the evidence suggests that these are one and the same emotion, with play constituting an offshoot of exploration in vertebrate evolution. Interest in some stimuli more than others occurs even in invertebrates and so preceded the evolution of exploratory behavior in vertebrates. We thus conclude that interest, exploration, and play constitute the same basic emotion, with exploration having evolved as a form of interest, followed by the evolution of play as an offshoot of exploration. Humor appreciation, or mirth, may have evolved as an offshoot of play; the primate origins of humor and similarities and differences between humor and play are described in Gray (2009) and Weisfeld (1993).

Such conclusions are ultimately judgment calls. Some theorists are partial to lengthy, minute category schemes ("splitters"), whereas others combine subcategories ("lumpers"). Perhaps the important thing is to include all relevant categories of emotional behavior, and do one's best to carve at the joints. 
Weisfeld, G. \& Cronin Weisfeld, C.: Is Play an Emotion? Some Ethological Observations Human Ethology Bulletin - Proc. of the V. ISHE Summer Institute (2016): 4-29

\section{CONCLUSION}

The study of play is relevant to various policy issues now being debated. These include the possibly excessive safety of playscapes, the amount of time devoted to adult-supervised play instead of peer-organized play, the need for recess, the decline in play in recent decades, the rise in computer play, the characteristics of mixed-sex play (Weisfeld, 1986; Weisfeld et al., 1982), and the advantages of mixed-age play (see Liu and LaFreniere, 2014). Gray (2009, 2011) has explored such issues at length. Clearly play is a behavior that cries out for observational research and the ethological approach. This behavior seems to have many benefits, consistent with its hedonic quality. Nature may know best how children should experience childhood; perhaps they should be left largely to their own devices, in the spirit of Rousseau.

\section{REFERENCES}

Aldis, O. (1975. Play fighting. New York: Academic Press. DOI

Alley, T. R., Brubaker, L. W., \& Fox O. M. (2013). Courtship feeding in humans? The effects of feeding versus providing food on perceived attraction and intimacy in dyads. Human Nature, 24, 430-443. DOI

Antonacci, D., Norscia, I., \& Palagi, E. (2010). Stranger to familiar: Wild stepsirhines manage xenophobia by playing. PLos One, 5 . DOI

Barners, J. L. , Bernstein, E., \& Bloom, P. (2015). Fact or fiction? Children's preferences for real versus make-believe stories. Imagination, Cognition and Personality, 34, 243-258. DOI

Bateson, P., \& Martin, P. (2013). Play, playfulness, creativity and innovation. Cambridge: Cambridge University Press. DOI

Berglund, H., Lindström, P., \& Savic, I. (2006). Brain response to putative pheromones in lesbian women. Proceedings of the National Academy of Sciences, 103, 8269-8274. DOI

Bernstein, I.S., \& Gordon, T. P. (1974). The function of aggression in primate societies. American Scientist, 62, 304-311.

Berridge, K. C.\& Robinson, T. E. (1998). What is the role of dopamine in reward: Hedonic impact, reward learning, or incentive salience? Brain Research Reviews, 28, 309-369. DOI

Blurton Jones, N.G. (1972). Categories of child interaction. In N.G. Blurton Jones (Ed.), Ethological studies of child behaviour (pp. 97-129). New York: Cambridge University Press.

Burghardt, G.M. (1988). Precocity, play, and the ectotherm-endotherm transition: Profound reorganization or superficial adaptation? In E.M. Blass (Ed.), Handbook of behavioral neurobiology. Vol. 9. Developmental psychobiology and behavioral ecology (pp. 107-148). New York: Plenum Press. DOI

Burghardt, G.M. (2005). The genesis of animal play: Testing the limits. Cambridge: MIT Press. 
Weisfeld, G. \& Cronin Weisfeld, C.: Is Play an Emotion? Some Ethological Observations Human Ethology Bulletin - Proc. of the V. ISHE Summer Institute (2016): 4-29

Byers, J. A., \& Walker, C. (1995). Refining the motor training hypothesis for the evolution of play. American Naturalist, 146, 25-40. DOI

Campos, J.J., \& Barrett, K.C. (1984). Toward a new understanding of emotions and their development. In C.E. Izard, J. Kagan \& R.B. Zajonc (Eds.), Emotions, cognition, and behavior (pp. 229-263). New York: Cambridge University Press.

Cannon, W. B. (1927). The James-Lange theory of emotions: A critical examination and an alternative theory. American Journal of Psychology, 39, 106-124. DOI

Chapman, H. A., Kim, D. A., Susskind, J. M., \& Anderson, A. K. (2009). In bad taste: Evidence for the oral origins of moral disgust. Science, 323, 1222-1226. DOI

Cheney, D. L., \& Seyfarth, R. M. (1990). How monkeys see the world: Inside the mind of another species. Chicago: University of Chicago Press.

Clark, C. D., \& Miller, P. J. (1998). Play. In H. Friedman (Ed.), Encyclopedia of mental health (Vol. 3) (pp. 189-197). San Diego: Academic Press.

Clarke, R., \& Glander, K. E. (1984). Female reproductive success in a group of free-ranging howling monkeys (Alouatta palliata) in Costa Rica. In M. F. Small (Ed.), Female primates: Studies by women primatologists (pp. 111-126). New York: Liss.

Clutton-Brock, T.H., \& Parker, G.A. (1995). Punishment in animal societies. Nature, 373, 209-216. $\underline{\text { DOI }}$

Cooke, B.M., Chowanadisai, W., \& Breedlove, S.M. (2000). Post-weaning social isolation of male rats reduces the volume of the medial amygdala and leads to deficits in adult sexual behavior. Behavioural Brain Research, 117, 107-113. DOI

Csikszentmihalyi, M., \& \& Hunter, J. (2003). Happiness in everyday life: The uses of experience sampling. Journal of Happiness Studies, 4, 185-199. DOI

Darwin, C. (1998). The expression of the emotions in man and animals (third edition). New York: Oxford University Press. DOI

deCatanzaro, D. A. (1999). Motivation and emotion: Evolutionary, physiological, developmental, and social perspectives. Upper Saddle River, NJ: Prentice-Hall.

Edwards, D.C. (1999). Motivation and Emotion: Evolutionary, physiological, cognitive, and social influences. London: Sage.

Eibl-Eibesfeldt, I. (1989). Human ethology. Hawthorne, NY: Aldine de Gruyter.

Ekman, P. (1994). All emotions are basic. In Ekman, P., \& Davidson, R. J., The nature of emotion: Fundamental questions (pp. 15-19).

Ekman, P., Davidson, R. J., \& Friesen, W. V. (1990). The Duchenne smile: Emotional expression and brain physiology II. Journal of Personality and social Psychology, 58, 342-353. DOI

Ekman, P., \& Friesen, W.V. (1971). Constants across cultures in the face and emotion. Journal of Personality and Social Psychology, 17, 124-129. DOI 
Weisfeld, G. \& Cronin Weisfeld, C.: Is Play an Emotion? Some Ethological Observations Human Ethology Bulletin - Proc. of the V. ISHE Summer Institute (2016): 4-29

Farb, N. A., Anderson, D. A., Mayberg, H., Bean, J., McKeon, D., \& Segal, Z. V. (2010). Minding one's emotions: Mindfulness training alters the neural expression of sadness. Emotion, 10, 2534. DOI

Fehr, E., \& Fischbacher, U. (2004). Third-party punishment and social norms. Evolution and Human Behavior, 25, 63-87. DOI

Flaherty, R. J. (1922). Nanook of the North (film).

Gaskins, S., Haight, W., \& Lancy, D. F. (2007). The cultural construction of play. In A. Göncü \& S. Gaskins (Eds.), Play and development: Evolutionary, sociocultural and functional perspectives (pp. 179-102). Hillsdale, NJ: Lawrence Erlbaum.

Geary, D. C. (2005). The origin of mind: Evolution of brain, cognition, and general intelligence. Washington, DC: American Psychological Association. DOI

Gray, P. (2009). Play as a foundation for hunter-gatherer social existence. American Journal of Play, 1, 476-522.

Gray, P. (2011). The decline of play and the rise of psychopathology in children and adolescents. American Journal of Play, 3, 443-463.

Hall, J. (1984). Nonverbal sex differences: Communication accuracy and expressive style. Baltimore: Johns Hopkins University Press.

Hamann, S. (2012). Mapping discrete and dimensional emotions onto the brain: Controversies and consensus. Trends in Cognitive Science, 16, 458-466. DOI

Harrison, N. A. Kreibig, S. D., \& Critchley, H. D. (2013). A two-way road: Efferent and afferent pathways of autonomic activity in emotion. In J Armony \& P. Vuilleumier (Eds.), The Cambridge handbook of human affective neuroscience (pp. 82-106). Cambridge: Cambridge University Press. DOI

Hayes, K. J., \& Hayes, C. (1951). The intellectual development of a home-raised chimpanzee. Proceedings of the American Philosophical Society, 95, 105-109.

Heinrich, B., \& Smolker, B. (1998). Play in common ravens (Corvus corax). In M. Bekoff \& J. A. Byers (Eds.), Animal play: Evolutionary, comparative and ecological perspectives (pp. 27-44). Cambridge: Cambridge University Press. DOI

Hutt, C., \& Hutt, S. J. (1965). Effects of environmental complexity on stereotyped behaviours of children. Animal Behaviour, 13, 1-4. DOI

Izard, C. E. (1977). Human emotions. New York: Plenum. DOI

Janson, C. H., \& van Schalk, C. P. (1993). Ecological risk aversion in juvenile primates: Slow and steady wins the race. In M. E. Pereira \& L. A. Fairbanks (Eds.), Juvenile primates: Life history, development, and behavior (pp. 57-76). New York: Oxford University Press.

Jensen, K., Call, J., \& Tomasello, M. (2007). Chimpanzees are vengeful, but not spiteful. Proceedings of the National Academy of Sciences, USA, 104, 13046-13050. DOI

Kleinginna, P. R. Jr., \& Kleinginna, A. M. (1981). A categorized list of emotion definitions with suggestions for a consensual definition. Motivation and Emotion, 5, 345-379. DOI 
Weisfeld, G. \& Cronin Weisfeld, C.: Is Play an Emotion? Some Ethological Observations Human Ethology Bulletin - Proc. of the V. ISHE Summer Institute (2016): 4-29

Komisaruk,B.R., \& Olds, J. (1968). Neuronal correlates of behavior in freely moving rats. Science, 161,810-812. DOI

Kuester, J., \& Paul, A. (1992). Influence of mate competition and female choice on male mating success in Barbary macaques. Behaviour, 120, 411-427. DOI

Kummer, H. (1971). Primate societies. Chicago: Aldine-Atherton.

LaFreniere, P. (2014a). Facial expressions, emotions and attachment in infancy. In P. LaFreniere and G. Weisfeld (Eds.), Evolutionary science of human behavior: an interdisciplinary approach (pp. 161-183). New York: Linus Books.

LaFreniere, P. (2014b). Play and childhood peer relations. In P. LaFreniere and G. Weisfeld (Eds.). Evolutionary science of human behavior: An interdisciplinary approach (pp. 185-203). New York: Linus Books.

Lang, P. J. (2010). Emotion and motivation: toward consensus definitions and a common research purpose. Emotion Review, 2, 229-233. DOI

Lee, P. C. (1984). Ecological constraints on the social development of vervet monkeys. Behaviour, 91, 245-262. DOI

Lillard, A.S., Lerner, M. D., Hopkins, E. J., Dore, R. A., Smith, E. D., \& Palmquist, C. M. (2013). The impact of pretend lay on children's development: A review of the evidence. Psychological Bulletin, 139, 1-34. DOI

Lindquist, K. A., Wager, T. D., Kober, H., Bliss-Moreau, E., \& Barrett, L. F. (2012). The brain basis of emotion> A meta-analytic review. Behavioral and Brain Sciences, 35, 121-143. DOI

Liu, C., \& LaFreniere, P. (2014). The effects of age-mixing on peer cooperation and competition. Human Ethology Bulletin, 29, 4-17.

McAlister, A., \& Peterson, C. (2006). A longitudinal study of child siblings and theory of mind development. Cognitive Development, 22, 258-270. DOI

McDougall, W. (1923). Outline of psychology. New York: Scribners.

McGrew, W. C. (2010). Chimpanzee technology. Science, 328, 579. DOI

Mofenson, H. C., \& Greensher, J. (1985). Management of the choking child. Pediatric Clinics of North America, 32, 183-192. DOI

Mohanty, A., \& Gottfried, J. A. (2013). Examining emotion perception and elicitation via olfaction. In J. Armony \& P. Vuilleumier (Eds.). The Cambridge handbook of human affective neuroscience (pp. 241-264). Cambridge: Cambridge University Press. DOI

Murphy, G. C., Nimmo-Smith, I., \& Lawrence, A. D. (2003). Functional neuroanatomy of emotions: a meta-analysis. Cognitive and Affective Behavioral Neuroscience, 3, 207-233. DOI

Neill, S.R. St.J. (1985), Rough-and-tumble and aggression in schoolchildren: Serious play? Animal Behaviour, 33, 1380-1382. DOI 
Weisfeld, G. \& Cronin Weisfeld, C.: Is Play an Emotion? Some Ethological Observations Human Ethology Bulletin - Proc. of the V. ISHE Summer Institute (2016): 4-29

Omark, D.R., Omark, M., \& Edelman, M.S. (1975). Formation of dominance hierarchies in young children: Action and perception. In T. Williams (E.), Psychological anthropology (pp. 87-107). The Hague: Mouton. DOI

Panksepp, J. (1994). Evolution constructed the potential for subjective experience within the neurodynamics of the mammalian brain. In P. Ekman \& R.J. Davidson (Eds.), The Nature of emotion: Fundamental questions (pp. 396-399). New York: Oxford University Press.

Panksepp, J. (1998). Affective neuroscience. Oxford: Oxford University Press.

Panksepp, J. (2005). Affective consciousness: Core emotional feelings in animals and humans. Consciousness and Cognition, 14, 30-80. DOI

Panksepp. J. (2008). The affective brain and core consciousness: How does neural activity generate emotional feelings? In M. Lewis, J. M. Haviland-Jones \& L. F. Barrett (Eds.), Handbook of emotions ( $3^{\text {rd }}$ ed.) (pp. 47-67). New York: Guilford Press.

Pellegrini, A. D., Horvat, M., \& Huberty, P. (1998). The relative cost of children's physical play. Animal Behaviour, 55, 1053-1061. DOI

Pellegrini, A.D., Huberty, P.D., \& Jones, I. (1995). The effects of recess timing on children's classroom and playground behavior. American Educational Research Journal, 32, 845-864. DOI

Pellegrini, A.D., \& Smith, P.K. (1998). Physical activity Play: the nature and function of a neglected aspect of play. Child Development, 69, 577-598. DOI

Pellis, S. M., \& Iwaniuk, A. N. (2000). Adult-adult play in primates: comparative analyses of tis origin, distribution, and evolution. Ethology, 106, 1083-1104. DOI

Phan, K. L., Wager, T., Taylor, S. F., \& Liberzon, I. (2002). Functional neuroanatomy of emotion: a meta-analysis of emotion activation studies in pet and fmri. Neuroimage, 16, 331-348. DOI

Piaget, J. (1932). The moral judgment of the child. New York: Harcourt Press. Reprinted version DOI

Plutchik, R. (1980). Emotion: A psychoevolutionary synthesis. New York: Harper and Row.

Provine, R. R., \& Yong, Y. L. (1991). Laughter: A stereotyped human vocalization. Ethology, 89, 115124. DOI

Pugh, G. E. (1977). The biological origin of human values. New York: Basic Books.

Rainville, P. (2013) Pain and the emotional responses to noxious stimuli. In J. Armony \& P. Vuilleumier (Eds.). The Cambridge handbook of human affective neuroscience (pp. 223-240). Cambridge: Cambridge University Press. DOI

Rivara, F. P., Bergman, A., B., LoFerfo, J. P., \& Weiss, W. (1982). Epidemiology of childhood injuries. II. Sex differences in injury rates. American Journal of Disabilities of Childhood, 136, 502-506. DOI

Roffman, I., Savage-Rumbaugh, S., Rubert-Pugh, E., Stadler, A., Ronen, A., \& Nevo, E. (2015). Preparation and use of varied natural tools for extractive foraging by bonobos (Pan paniscus). American Journal of Physical Anthropology, 158, 78-91. DOI 
Weisfeld, G. \& Cronin Weisfeld, C.: Is Play an Emotion? Some Ethological Observations Human Ethology Bulletin - Proc. of the V. ISHE Summer Institute (2016): 4-29

Roper, R., \& Hinde, R.A. (1978). Social play in a play group: Consistency and complexity. Child Development, 49, 570-579. DOI

Sacks, J. J., Smith, J. D., Kaplan, K. M., Lambert, D. A., Stattin, R. W., \& Sikes, R. K. (1989). The epidemiology of injuries in Atlanta day care centers. Journal of the American Medical Association, 262, 1641-1645. DOI

Sander, D. (2013). Models of emotion: The affective neuroscience approach. In J. Armony \& P. Vuilleumier (Eds.). The Cambridge handbook of human affective neuroscience (pp. 5-53). Cambridge: Cambridge University Press. DOI

Scherer, K. R. (1993). Neuroscience projections to current debates in emotion psychology. Cognition and Emotion. 7, 1-41. DOI

Schmehl, S., \& Oberzaucher, L. (2014). Multimodal communication. In P. LaFreniere \& G. Weisfeld (Eds.), Evolutionary science of human behavior: An interdisciplinary approach (pp. 231-255). Ronkonkoma, NY: Linus Learning.

Schneider, G.E. (2014). Brain structure and its origins. Cambridge: MIT Press.

Singer, D. G., Singer, J. L, D’Augustino, H., \& DeLong, R. (2009). Children's pastimes and play in sixteen nations: Is free play declining? American Journal of Play, 1, 283-312.

Smith, P.K. (Ed.) (1984). Play in animals and humans. Oxford: Basic Blackwell.

Smith, P.K. (2010). Children and play: Understanding children's worlds. Malden, MA: Wiley.

Smith, P.K. (2015). Does play matter: Arguments about the role of play in animals and humans. Plenary talk presented at the Summer Institute of the International Society for Human Ethology, Athens.

Smith, P.K., \& Connolly, K. (1980). The ecology of preschool behaviour. Cambridge; Cambridge University Press.

Smoll, F. L., \& Schutz, R. W. (1985). Physical fitness differences between athletes and nonathletes: Do changes occur as a function of age and sex? Human Movement Science, 4, 189-202. DOI

Tanner, J.M. (1970). Physical growth. In P.H. Mussen (Ed.) Carmichael's manual of child psychology ( $3^{\text {rd }}$ ed.) (pp. 77-155). New York: Wiley.

Thelen, E. (1995). Motor development: A new synthesis. American Psychologist, 50, 79-95. DOI

Tinbergen, N. (1963). On the aims and methods of ethology. Zeitgeist für Tierpsychologie, 20, 410433. DOI

Tracy, J.L., \& Robins, R.W. (2008). The nonverbal expression of pride: Evidence for cross-cultural recognition. Journal of Personality and Social Psychology, 94, 516-530. DOI

Trivers, R.L. (1971). The evolution of reciprocal altruism. Quarterly Review of Biology, 46, 35-57. DOI

Weisfeld, C.C. (1986). Female behavior in mixed-sex competition: A review of the literature. Developmental Review, 6, 278-299. DOI 
Weisfeld, G. \& Cronin Weisfeld, C.: Is Play an Emotion? Some Ethological Observations Human Ethology Bulletin - Proc. of the V. ISHE Summer Institute (2016): 4-29

Weisfeld, C.C., Weisfeld, G. E., \& Callaghan, J.W. (1982). Female inhibition in mixed-sex competition among young adolescents. Ethology and Sociobiology, 3, 29-42. DOI

Weisfeld, G.E. (1972). Violations of social norms as inducers of aggression. International Journal of Group Tensions, 2, 53-70.

Weisfeld, G. E. (1993). The adaptive value of humor and laughter. Ethology and Sociobiology, 14, 141-169. DOI

Weisfeld, G.E., Muczenski, D.M., Weisfeld, C.C., \& Omark, D.R. (1987). Stability of boys' social success over an eleven-year period. In J.A. Meacham (Ed.), Interpersonal relations: Family peers, friends (pp 58-80). Basel: Karger. DOI

Weisfeld, G. E., Weisfeld, C. C., \& Callaghan, J. W. (1984). Peer and self perceptions in Hopi and Afro-American third- and sixth-graders. Ethos, 12, 64-84. DOI

Zajonc, R. B. (1984). On primacy of affect. In K. R. Scherer \& P. Ekman (Eds.), Approaches to emotion (pp. 259-270). Hillsdale, NJ: Lawrence Erlbaum. 